\title{
Bose-Einstein condensation and symmetry breaking
}

\author{
András Sütő \\ Research Institute for Solid State Physics and Optics \\ Hungarian Academy of Sciences \\ P. O. Box 49, H-1525 Budapest \\ Hungary \\ E-mail: suto@szfki.hu
}

\begin{abstract}
Adding a gauge symmetry breaking field $-\nu \sqrt{V}\left(a_{0}+a_{0}^{*}\right)$ to the Hamiltonian of some simplified models of an interacting Bose gas we compute the condensate density and the symmetry breaking order parameter in the limit of infinite volume and prove Bogoliubov's asymptotic hypothesis $\lim _{V \rightarrow \infty}\left\langle a_{0}\right\rangle / \sqrt{V}=\operatorname{sgn} \nu \lim _{V \rightarrow \infty} \sqrt{\left\langle a_{0}^{*} a_{0}\right\rangle / V}$ where the averages are taken in the ground state or in thermal equilibrium states. Letting $\nu$ tend to zero in this equation we obtain that Bose-Einstein condensation occurs if and only if the gauge symmetry is spontaneously broken. The simplification consists in dropping the off-diagonal terms in the momentum representation of the pair interaction. The models include the mean field and the imperfect (Huang-Yang-Luttinger) Bose gas. An implication of the result is that the compressibility sum rule cannot hold true in the ground state of the one-dimensional mean-field Bose gas. Our method is based on a resolution of the Hamiltonian into a family of single-mode $(\mathbf{k}=0)$ Hamiltonians and on the analysis of the associated microcanonical ensembles.
\end{abstract}

PACS: 03.75.Hh, 05.30.Jp

\section{INTRODUCTION AND RESULTS}

The relation between Bose-Einstein condensation (BEC) and a spontaneous breakdown of the gauge symmetry of the system (shortly, symmetry breaking) is not entirely understood. Symmetry breaking is absent in textbook discussions of BEC of the ideal Bose gas and does not appear in the general definition of BEC by Penrose and Onsager [1] either. It is, on the other hand, a central notion of Bogoliubov's theory of superfluidity and superconductivity, dealt with extensively under the name of quasi-averaging [2]. Bose-Einstein condensation is the accumulation of a macroscopic number of particles in a single-particle state. In homogenous (translation invariant) situation this state is $\varphi_{0}(\mathbf{r}) \equiv e^{i \phi} / \sqrt{V}$ where $V$ is the volume of the cube on which the system is defined with periodic boundary conditions and $\phi$ is a real constant. The macroscopic occupation of $\varphi_{0}$ means that the thermal (or ground state) average of its occupation number operator $N_{0}$ increases proportionally to $V$ as $V$ tends to infinity, which can be shown to be equivalent to an off-diagonal long-range order (ODLRO). Hamiltonians conserving particle number possess a continuous gauge symmetry related to the phase $\phi$. For pair interactions a general Hamiltonian reads

$$
H=\sum \varepsilon(\mathbf{k}) N_{k}-\mu N+\frac{1}{2 V} \sum_{\mathbf{q}, \mathbf{k}, \mathbf{k}^{\prime}} v(\mathbf{q}) a_{\mathbf{k}+\mathbf{q}}^{*} a_{\mathbf{k}^{\prime}-\mathbf{q}^{\mathbf{q}}} a_{\mathbf{k}^{\prime}} a_{\mathbf{k}}
$$

where $\varepsilon(\mathbf{k})=\hbar^{2} \mathbf{k}^{2} / 2 m, \mu$ and $v(\mathbf{q})=v(-\mathbf{q})$ are real numbers, $N_{\mathbf{k}}=a_{\mathbf{k}}^{*} a_{\mathbf{k}}, N=\sum N_{\mathbf{k}}, a_{\mathbf{k}}^{*}$ creates a boson in the single-particle state $\varphi_{\mathbf{k}}(\mathbf{r})=(1 / \sqrt{V}) \exp \{i \mathbf{k r}\}$ and $\mathbf{q}, \mathbf{k}, \mathbf{k}^{\prime}$ are allowed wave vectors, i.e. compatible with the periodic boundary condition. Averaging any $a_{\mathbf{k}}$ with eigenstates or the density matrix generated by $H$ yields zero. (We disregard a possible accidental degeneracy of eigenstates belonging to different particle numbers.) For any real $\phi$ changing all $a_{\mathbf{k}}$ simultaneously into $e^{-i \phi} a_{\mathbf{k}}$ (and $a_{\mathbf{k}}^{*}$ into $e^{i \phi} a_{\mathbf{k}}^{*}$ ) leaves the commutation relations and the Hamiltonian invariant. This gauge symmetry can be broken by adding a term which breaks particle number conservation, e.g. for real $\nu$ and $\phi$ the operators $H-\nu \sqrt{V}\left(e^{-i \phi} a_{0}+e^{i \phi} a_{0}^{*}\right)$ are no more gauge invariant. Still they are unitary equivalent for different $\phi$, therefore $\phi=0$ can be taken without restricting generality. Now the thermal or ground state average $\left\langle a_{0}\right\rangle$ is nonvanishing, and we speak about a spontaneous breakdown of the gauge symmetry if the 'quasi-average'

$$
\lim _{\nu \rightarrow 0} \lim _{V \rightarrow \infty} \frac{1}{\sqrt{V}}\left\langle a_{0}\right\rangle \neq 0
$$

When this occurs, we are dealing with a continuum of infinite volume pure states, each characterized by a phase $\phi$, and the state we obtain by fixing $\nu=0$ in finite volumes and taking the limit $V \rightarrow \infty$ is the uniform mixture of pure states with $0 \leq \phi<2 \pi$.

By now the physical reality of symmetry breaking at least in a non-translation-invariant situation - has become an experimental fact, manifesting itself in the interference of two condensates of different phases [3]. It is all the more embarrassing that there seems to exist no theoretical proof of its occurrence. There is Hohenberg's theorem on the absence of symmetry breaking in one and two dimensional Bose gases at positive temperatures [46 . In some other cases one can prove BEC by showing either the existence of ODLRO or the macroscopic occupation of a one-particle state. An example for the first 
is the hard-core Bose lattice gas at half-filling [7], for the second the trapped Bose gas [8]. However, the Schwarz inequality

$$
\left|\left\langle a_{0}\right\rangle\right| \leq \sqrt{\left\langle N_{0}\right\rangle}
$$

indicates that the absence of symmetry breaking does not automatically imply the absence of $\mathrm{BEC}$, and vice versa, BEC does not obviously imply symmetry breaking. A closely related question is the validity of Bogoliubov's approximation [9] which consists in replacing $a_{0}$ and $a_{0}^{*}$ by complex numbers $c$ resp. $c^{*}$. A partial answer was given by Ginibre a long time ago [10]. Ginibre considered this replacement in the Hamiltonian or in the grand-canonical density matrix, with or without the symmetry breaking field, and proved that in all cases by choosing $c$ so that it maximizes the approximate pressure in finite volumes the result converges to the exact pressure in the limit of infinite volume. This suggests that symmetry breaking indeed takes place when there is BEC, and the inequality (1.2) saturates asymptotically.

In this paper we will prove that for any $\nu \neq 0$,

$$
\lim _{V \rightarrow \infty}\left\langle a_{0}\right\rangle / \sqrt{V}=\operatorname{sgn} \nu \lim _{V \rightarrow \infty} \sqrt{\left\langle N_{0}\right\rangle / V}
$$

in the ground state and thermal equilibrium states of the mean-field Bose gas and of other models whose interaction is diagonal in momentum representation. The corresponding Hamiltonians are obtained from (1.1) by dropping the off-diagonal terms:

$$
\begin{array}{r}
H^{\mathrm{FD}}=\sum \varepsilon(\mathbf{k}) N_{k}-\mu N+\frac{v_{0}}{2 V}\left(N^{2}-N\right) \\
+\frac{1}{2 V} \sum_{\mathbf{k} \neq \mathbf{k}^{\prime}} v\left(\mathbf{k}^{\prime}-\mathbf{k}\right) N_{\mathbf{k}} N_{\mathbf{k}^{\prime}} .
\end{array}
$$

Here $v_{0}=v(0)>0$, and for the sake of simplicity we consider only $v(\mathbf{k}) \geq 0$. In the mean-field model, $H^{\mathrm{MF}}$, $v(\mathbf{k})=0$ for $\mathbf{k} \neq 0$. The imperfect Bose gas is defined by keeping the diagonal part of the $\delta$ interaction, so that $v(\mathbf{k})=v_{0}$ for all $\mathbf{k}[11]$. Note that only the interaction of the mean-field Bose gas is diagonal in coordinate representation. The other models may present nonintuitive features like anomalies in the spectrum of elementary excitations, see below, and the Thouless effect, a jump-discontinuity in the condensate density as the chemical potential increases $[12,13]$. The thermal equilibrium properties of the full diagonal model (1.4) were rigorously studied by Dorlas et al. [13].

The ground state of each Hamiltonian of the family (1.4) in the Fock space is $\left|N=N_{0}=m_{V}\right\rangle$ where $m_{V}$ is the nonnegative integer closest to $\mu V / v_{0}+\frac{1}{2}$. In the limit of infinite volume the ground state energy density is $-\left(v_{0} / 2\right) \lim \left(m_{V} / V\right)^{2}$. We will study the Hamiltonian

$$
H_{\nu}^{\mathrm{FD}}=H^{\mathrm{FD}}-\nu \sqrt{V}\left(a_{0}+a_{0}^{*}\right)
$$

and prove that for $\nu \neq 0$ the ground state energy density

$$
\begin{aligned}
e_{0} \equiv \lim _{V \rightarrow \infty} E_{0} / V & =\min _{s \geq 0}\left\{v_{0} s^{2} / 2-\mu s-2|\nu| \sqrt{s}\right\} \\
& =v_{0} s_{0}^{2} / 2-\mu s_{0}-2|\nu| \sqrt{s_{0}},
\end{aligned}
$$

the order parameter

$$
\alpha_{0} \equiv \lim _{V \rightarrow \infty} \frac{1}{\sqrt{V}}\left\langle a_{0}\right\rangle=\operatorname{sgn} \nu \sqrt{s_{0}}
$$

and the condensate density

$$
\rho_{0} \equiv \lim _{V \rightarrow \infty} \frac{1}{V}\left\langle N_{0}\right\rangle=s_{0}=\alpha_{0}^{2} .
$$

In (1.7) and (1.8) the averages are taken in the ground state of $H_{\nu}^{\mathrm{FD}}$. The minimizer $s_{0}$ coincides with the full particle density. It is positive for any value of $\mu$, but goes to zero with a vanishing $\nu$ if $\mu \leq 0$. The extension of Eq. (1.3) to positive temperatures will be obtained by proving the analog of Eqs. (1.6)-(1.8) for the ground state of certain microcanonical ensembles and by referring to the equivalence of micro- and grand-canonical ensembles. Equation (1.3) is not a statement about the existence of BEC. It holds even if the chemical potential is below its critical value when both sides vanish as $\nu$ tends to zero. It however guaranties that BEC and a spontaneous breakdown of the gauge symmetry occur simultaneously.

The result for the ground state is independent of the dimension, although $H^{\mathrm{FD}}$ depends on it. In particular, there is symmetry breaking along with the Bose condensation in the ground state of the one-dimensional meanfield Bose gas. Because of the finite compressibility of this system, our finding is in conflict with an earlier argument according to which there should be no symmetry breaking in the ground state of one-dimensional interacting Bose gases [14]. To track down the source of this contradiction and also by a general interest we consider here some implications of the results listed above on the properties of the excitation spectrum. The nonrelativistic version of the Goldstone theorem states that a spontaneously broken continuous symmetry implies the existence of gapless excitations, i.e. eigenstates whose energy tends to zero with a vanishing momentum. Wagner's analysis [15] based on the Bogoliubov inequality and on Bogoliubov's $1 / q^{2}$-theorem concluded that the nature of the long-wavelength excitations was uncorrelated with symmetry breaking and was governed by the range of the interaction. Our conclusion for this particular family of interactions is similar. As it is shown below, there are gapless excitations in the mean-field model whose interaction is of infinite range but there is a gap to excitations with a non-vanishing momentum in the spectrum of the other models. This seems to lead to another contradiction. In the ground state version of the $1 / q^{2}$-theorem,

$$
\left\langle N_{\mathbf{q}}\right\rangle \geq \frac{1}{4} \frac{\left(E_{\mathbf{q}}-E_{0}\right)\left|\left\langle a_{0}\right\rangle\right|^{2}}{\varepsilon(\mathbf{q})\langle N\rangle+\left|\nu\left\langle a_{0}\right\rangle\right| \sqrt{V}}-\frac{1}{2},
$$

$E_{0}$ is the ground state energy and $E_{\mathbf{q}}$ is the energy of the lowest lying eigenstate of momentum $\hbar \mathbf{q}$ of the symmetry breaking Hamiltonian [16]. In the actual case (1.9) can be made stronger. The Hamiltonian $H_{\nu}^{\mathrm{FD}}$ commutes separately with each $N_{\mathbf{k}}, \mathbf{k} \neq 0$. We shall prove that 
for $\nu \neq 0$ the ground state is still in the invariant subspace $\left\{N_{\mathbf{k}}=0 \mid \mathbf{k} \neq 0\right\}$. Then the ground state average $\left\langle N_{\mathbf{q}}\right\rangle=0$ and from the derivation of Eq. (1.9) one sees that $E_{\mathbf{q}}$ can be taken to be the smallest eigenvalue among the eigenstates with $N_{\mathbf{q}}=1$ and $N_{\mathbf{k}}=0$ if $\mathbf{k} \neq 0$ or $\mathbf{q}$, i.e. a single particle carrying the total momentum, which can be much greater than the minimum eigenvalue among eigenstates of momentum $\hbar \mathbf{q}$. In general, summing (1.9) over allowed $\mathbf{q}$ such that $0<|\mathbf{q}|<q_{0}$, dividing by $V$ and letting $V$ tend to infinity while keeping $q_{0}$ fixed, we arrive at

$$
\begin{aligned}
\rho-\rho_{0} & \geq \frac{1}{2(2 \pi)^{d}} \\
& \times \int_{|\mathbf{q}|<q_{0}}\left(\frac{1}{2} \frac{\left|\alpha_{0}\right|^{2} \lim _{V \rightarrow \infty}\left(E_{\mathbf{q}}-E_{0}\right)}{\varepsilon(\mathbf{q}) \rho+\left|\nu \alpha_{0}\right|}-1\right) \mathrm{d} \mathbf{q}
\end{aligned}
$$

where $\rho$ is the total particle density and $d$ is the dimension [17]. In the actual case $\rho=\rho_{0}=\left|\alpha_{0}\right|^{2}$. In Section IV we will derive the gap to one-particle excitations,

$$
\begin{array}{r}
E_{\mathbf{q}}-E_{0}=\varepsilon(\mathbf{q})+\mu \\
\frac{v(\mathbf{q})}{v_{0}}+|\nu| \frac{v_{0}+v(\mathbf{q})}{\sqrt{\mu v_{0}}} \\
+O\left(\nu^{2}\right)+O(1 / V),
\end{array}
$$

valid for $\mu>0$. The finite-size correction $\sim 1 / V$ is uniform in $\nu$ so that the limits $V \rightarrow \infty$ and $\nu \rightarrow 0$ are interchangeable. We can use this result in (1.10) and see what we obtain when $\nu$ goes to zero. For the mean-field model $\lim _{\nu \rightarrow 0} \lim _{V \rightarrow \infty}\left(E_{\mathbf{q}}-E_{0}\right)=\varepsilon(\mathbf{q})$, therefore (1.10) holds true. For the other models the gap implies a diverging integral in one and two dimensions and, thus, a violation of the inequality (1.10). The cause of the contradiction is our misuse of (1.9), which cannot be applied to $H_{\nu}^{\mathrm{FD}}$ if $v(\mathbf{k}) \neq 0$ for some $\mathbf{k} \neq 0$ : These interactions are non-diagonal (nonlocal) in coordinate representation and therefore do not commute with $\rho_{\mathbf{k}}$, the Fourier transform of the density operator, which is diagonal. This invalidates the $f$-sum rule [18], used in the derivation of Eq. (1.9). Turning to the controversy in connection with the mean-field model, we recall an improved version of (1.9) due to Pitaevskii and Stringari [14],

$$
\left\langle N_{\mathbf{q}}\right\rangle \geq \frac{1}{4} \frac{\left|\left\langle a_{0}\right\rangle\right|^{2}}{\left\langle\rho_{-\mathbf{q}} \rho_{\mathbf{q}}\right\rangle}-\frac{1}{2}=\frac{1}{4} \frac{\left|\left\langle a_{0}\right\rangle\right|^{2}}{\langle N\rangle S(\mathbf{q})}-\frac{1}{2}
$$

where $S(\mathbf{q})$ is the static structure function. This formula is valid for all the models discussed in this paper and leads to

$$
\int_{|\mathbf{q}|<q_{0}}\left(\frac{1}{2 S(\mathbf{q})}-1\right) \mathrm{d} \mathbf{q} \leq 0 .
$$

We can conclude that $q^{d} / S(\mathbf{q}) \rightarrow 0$ as $q \rightarrow 0$ in all models considered here. In the general case, when the $f$-sum rule holds true, (1.9) can be obtained also from (1.12). When both the $f$-and the compressibility sum rules [18] hold,

$$
\lim _{q \rightarrow 0} q / S(\mathbf{q}) \geq \sqrt{4 m / \hbar^{2} \kappa \rho},
$$

where $\kappa$ is the ground state compressibility. In the meanfield Bose gas $\kappa=1 / v_{0} \rho^{2}<\infty$. Thus, (1.14) cannot hold true in one dimension and because the $f$-sum rule is valid, the compressibility sum rule must fail in the ground state of the one-dimensional mean-field Bose gas.

Note that the positive temperature variant of inequality (1.9), with $2 k_{B} T$ replacing $E_{\mathbf{q}}-E_{0}$, cf. [2, 16], also relies on the commutability of $\rho_{\mathbf{k}}$ with the interaction. Thus, it cannot be applied to prove the absence of symmetry breaking for $H^{\mathrm{FD}}$ in one and two dimensions at $T>0$ if $v(\mathbf{k})$ is nonvanishing for nonzero k. Rightly so, because in these systems, e.g. in the imperfect Bose gas [11], there can be BEC at $T>0$ even in one dimension, see also $[12,13]$.

All our results are obtained by representing the Hamiltonian as a Jacobi matrix in microcanonical ensembles or, equivalently, by studying some tight-binding Schrödinger equations on the spectrum of $N_{0}$. In Section II we show that $N_{\mathbf{k}}=0$ for all $\mathbf{k} \neq 0$ in finite volume ground states. Formulas (1.6)-(1.8) will be derived in Section III. The analog of (1.6) for the ground state of microcanonical ensembles is presented there, that of (1.7) and (1.8) in Section IV which also contains the derivation of Eq. (1.11). In Section $\mathrm{V}$ we discuss the extension of the results to positive temperatures. The paper ends with a Summary.

\section{GROUND STATE IN FINITE VOLUMES}

The Hamiltonians commute with each $N_{\mathbf{k}}, \mathbf{k} \neq 0$. One can therefore decompose them into operators acting on invariant subspaces with fixed $N_{\mathbf{k}}$. Let $\mathbf{n}=\left\{n_{\mathbf{k}}\right\}_{\mathbf{k} \neq 0}$ denote terminating sequences of nonnegative integers, i.e. $\|\mathbf{n}\|=\sum_{\mathbf{k} \neq 0} n_{\mathbf{k}}<\infty$. Then

$$
H_{\nu}^{\mathrm{FD}}=\oplus_{\mathbf{n}} H(\mathbf{n})
$$

where $H(\mathbf{n})$ is the restriction of $H_{\nu}^{\mathrm{FD}}$ onto the subspace $\left\{N_{\mathbf{k}}=n_{\mathbf{k}} \mid \mathbf{k} \neq 0\right\}$. Because $v_{0}>0, H_{\nu}^{\mathrm{FD}}$ has a discrete point spectrum with $+\infty$ as the only accumulation point, $\operatorname{Tr} \exp \left\{-\beta H_{\nu}^{\mathrm{FD}}\right\}<\infty$ for $\beta>0$, and the same holds for each $H(\mathbf{n})$. In this section we prove the following assertion.

In any finite volume the ground state of $H_{\nu}^{\mathrm{FD}}$ is in the subspace $\left\{N_{\mathbf{k}}=0 \mid \mathbf{k} \neq 0\right\}$, i.e. it is the ground state of $H(0)$.

This property is specific to the diagonal models and $v(\mathbf{k}) \geq 0$. A general interaction does not commute with $N_{\mathrm{k}}$. This knowledge about the ground state can but need not be used for the derivation of the formulas (1.6), (1.7) and (1.8). It plays, however, a role in the interpretation and computation of $E_{\mathbf{q}}-E_{0}$ in Eq. (1.11).

One has to prove that for each $\mathbf{n} \neq 0$ the ground state energies of $H(\mathbf{n})$ and $H(0)$ satisfy the inequality $E_{0}[H(\mathbf{n})]>E_{0}[H(0)]$. This is immediately seen if $\|\mathbf{n}\| \geq 1+2 \mu V / v_{0}$. The operator $H(\mathbf{n})-H(0)$ is diagonal and in this case it has positive elements, which 
implies the result. In the case $\|\mathbf{n}\|<1+2 \mu V / v_{0}$ (which can occur only if $\mu>0$ ), however, it may have both positive and negative elements and we need a more elaborate argument. We shall interpose between $H(\mathbf{n})$ and $H(0)$ three auxiliary operators, $K(\|\mathbf{n}\|), K(\|\mathbf{n}\|, 0)$ and $K(\|\mathbf{n}\|, 1)$ and show the following order of ground state energies:

$$
\begin{aligned}
E_{0}[H(\mathbf{n})]>E_{0}[K(\|\mathbf{n}\|)]=E_{0}[K(\|\mathbf{n}\|, 0)] \\
>E_{0}[K(\|\mathbf{n}\|, 1)] \geq E_{0}[H(0)] .
\end{aligned}
$$

Define

$$
K(p)=\frac{v_{0}}{2 V}\left(N_{0}+p\right)^{2}-\left(\mu+\frac{v_{0}}{2 V}\right)\left(N_{0}+p\right)-\nu \sqrt{V}\left(a_{0}+a_{0}^{*}\right)
$$

where $p$ is a real number. In particular, $K(0)=H(0)$. The operator inequality

$$
H(\mathbf{n})>K(\|\mathbf{n}\|)
$$

holds true for $\mathbf{n} \neq 0$, because $H(\mathbf{n})-K(\|\mathbf{n}\|)$ is a diagonal operator with entries $\geq \sum \varepsilon(\mathbf{k}) n_{\mathbf{k}}>0$. For the ground state energies (2.4) implies

$$
E_{0}[H(\mathbf{n})]>E_{0}[K(\|\mathbf{n}\|)] .
$$

Let $p$ be a nonnegative integer. Using the basis $\left\{\left|N_{0}=m\right\rangle\right\}_{m=0}^{\infty}$ of $N_{0}$-eigenstates, $K(p)$ can be represented by a semi-infinite tridiagonal matrix (Jacobi matrix), denoted also by $K(p)$. The diagonal matrix elements satisfy $K(p)_{m m}=K(0)_{m+p, m+p}$ for $m \geq$ 0 . The off-diagonal elements are the same for all $p$, $K(p)_{m-1, m}=K(p)_{m, m-1}=-\nu \sqrt{m V}$ if $m \geq 1$, and 0 otherwise. The matrices $K(p)$ act in the Hilbert space $\ell_{2}(\mathbb{N})=\left\{\left.(\psi(m))_{0}^{\infty}\left|\sum_{m}\right| \psi(m)\right|^{2}<\infty\right\}$. We extend them without changing the notation onto $\ell_{2}(\mathbb{Z})=$ $\left\{\left.(\psi(m))_{-\infty}^{\infty}\left|\sum_{m}\right| \psi(m)\right|^{2}<\infty\right\}$ by setting $K(p)_{m n}=0$ if either $m$ or $n$ is negative. Then $\ell_{2}(\mathbb{N})$ and $\ell_{2}(\mathbb{Z} \backslash \mathbb{N})$ are invariant subspaces of each $K(p)$ which acts on the first space as the original operator and on the second as the null operator. If $E_{0}[K(p)]<0$, the ground state energy of the extended operator is the same as that of the original one. Let $U_{p}$ be the left shift by $p$, i.e. for $\psi \in \ell_{2}(\mathbb{Z})$, $\left(U_{p} \psi\right)(m)=\psi(m+p)$. Then

$$
\begin{aligned}
& \left(U_{p}^{-1} K(p) U_{p}\right)_{m m}=K(p)_{m-p, m-p} \\
& =\left\{\begin{array}{cll}
0 & , & m \leq p-1 \\
K(0)_{m m} & , & m \geq p
\end{array}\right.
\end{aligned}
$$

and

$$
\begin{aligned}
& \left(U_{p}^{-1} K(p) U_{p}\right)_{m-1, m}=K(p)_{m-1-p, m-p} \\
& \quad=\left\{\begin{array}{cl}
0 & m \leq p \\
-\nu \sqrt{(m-p) V}, & m \geq p+1
\end{array} .\right.
\end{aligned}
$$

Define $K(p, 0)=U_{p}^{-1} K(p) U_{p}$. The operator $U_{p}$ is unitary, thus $E_{0}[K(p, 0)]=E_{0}[K(p)]$.

For the definition of $K(p, 1)$ we split the matrices $K(p, 0)$ and $K(0)$ into diagonal $D$ and off-diagonal $Q$ parts, $K(p, 0)=D(p)+Q(p)$ and $K(0)=D(0)+Q(0)$, and set $K(p, 1)=D(p)+Q(0)$. Furthermore, we introduce an interpolating matrix function

$$
K(p, t)=t K(p, 1)+(1-t) K(p, 0)=D(p)+t[Q(0)-Q(p)]
$$

By the Hellmann-Feynman theorem

$$
\begin{aligned}
& \frac{\partial}{\partial t} E_{0}[K(p, t)]=\left(\psi_{t},[Q(0)-Q(p)] \psi_{t}\right) \\
& =\sum_{m, n \geq 0} \psi_{t}(m)[Q(0)-Q(p)]_{m n} \psi_{t}(n)
\end{aligned}
$$

where $\psi_{t}$ is the normalized ground state of $K(p, t)$. Choose $\nu$ to be positive (changing the sign of $\nu$ amounts to a unitary transformation with a diagonal matrix of elements $\left.(-1)^{m}\right)$. Then

$$
[Q(0)-Q(p)]_{m-1, m}=[Q(0)-Q(p)]_{m, m-1}<0
$$

for $m \geq 1$ implying (through the variational principle or the Perron-Frobenius theorem) $\psi_{t}(m)>0$ for $m \geq$ 0 . Thus the derivative (2.9) is negative, which proves $E_{0}[K(p, 0)]>E_{0}[K(p, 1)]$.

At last, we note that $K(p, 1)-K(0)=D(p)-D(0) \geq 0$ if $p=\|\mathbf{n}\| \leq 1+2 \mu V / v_{0}$ because

$[D(p)-D(0)]_{m m}=\left\{\begin{array}{cl}-K(0)_{m m} \geq 0 & , \quad 0 \leq m \leq p-1 \\ 0 & , \quad \text { otherwise }\end{array}\right.$

Therefore $E_{0}[K(\|\mathbf{n}\|, 1)] \geq E_{0}[K(0)]$ indeed. Here $E_{0}[K(0)]$ is the ground state energy of the extended operator. If $E_{0}[H(0)]$ was positive, we would obtain $E_{0}[K(0)]=0$. However, $E_{0}[H(0)]<E_{0}\left[H^{\mathrm{FD}}\right]<0$ can be seen by using $\left|N=N_{0}=m_{V}\right\rangle$ as a variational wave function, so that $E_{0}[K(0)]=E_{0}[H(0)]$.

\section{GROUND STATE IN INFINITE VOLUME}

Our aim in this section is to derive Eqs. (1.6)-(1.8). Incidentally, we shall obtain the ground state energy density for $H(\mathbf{n})$, cf. (2.1).

Each $H(\mathbf{n})$ is an unbounded operator which is bounded below. A common lower bound is that of $H_{\nu}^{\mathrm{FD}}$,

$$
\begin{aligned}
H_{\nu}^{\mathrm{FD}} & \geq H^{\mathrm{FD}}-|\nu|\left(V+N_{0}\right) \\
& \geq-\left[\frac{v_{0}}{2}\left(\frac{\mu+|\nu|}{v_{0}}+\frac{1}{2 V}\right)^{2}+|\nu|\right] V .
\end{aligned}
$$

Here we have made use of $\pm \sqrt{V}\left(a_{0}+a_{0}^{*}\right) \leq V+N_{0}$ which comes from $\left(\sqrt{V} \pm a_{0}^{*}\right)\left(\sqrt{V} \pm a_{0}\right) \geq 0$.

To obtain a good variational upper bound on $E_{0}[H(\mathbf{n})]$, note that $H^{\mathrm{FD}}$ of Eq. (1.4) has a decomposition analogous to $(2.1), H^{\mathrm{FD}}=\oplus_{\mathbf{n}} H_{\text {diag }}(\mathbf{n})$ where $H_{\text {diag }}(\mathbf{n})$ is the diagonal part of $H(\mathbf{n})$. Let $\left|N_{0}=J(\mathbf{n})\right\rangle$ be the ground state of $H_{\operatorname{diag}}(\mathbf{n})\left(J(0)=m_{V}\right)$. Then with

$$
\psi=V^{-1 / 4} \sum_{m=J(\mathbf{n})}^{J(\mathbf{n})+\sqrt{V}}\left|N_{0}=m\right\rangle
$$


as a variational wave function we obtain

$$
E_{0}[H(\mathbf{n})] \leq E_{0}\left[H_{\operatorname{diag}}(\mathbf{n})\right]-2|\nu| \sqrt{J(\mathbf{n}) V}+O(\sqrt{V}) .
$$

This upper bound will turn out to be the precise asymptotic result up to order $|\nu|$.

Let us introduce

$$
\begin{gathered}
x_{V}(\mathbf{n})=\frac{1}{V} \sum_{\mathbf{k} \neq 0} n_{\mathbf{k}} \quad y_{V}(\mathbf{n})=\frac{1}{V} \sum_{\mathbf{k} \neq 0} v(\mathbf{k}) n_{\mathbf{k}} \\
z_{V}(\mathbf{n})=\frac{1}{V} \sum \varepsilon(\mathbf{k}) n_{\mathbf{k}}+\frac{1}{2 V^{2}} \sum_{0 \neq \mathbf{k} \neq \mathbf{k}^{\prime} \neq 0} v\left(\mathbf{k}^{\prime}-\mathbf{k}\right) n_{\mathbf{k}} n_{\mathbf{k}^{\prime}}
\end{gathered}
$$

All three are real nonnegative numbers, vanishing if $\|\mathbf{n}\|=0$. With them

$$
\begin{array}{r}
H(\mathbf{n}) / V \equiv h_{V}\left(x_{V}, y_{V}, z_{V}\right) \\
=\frac{v_{0}}{2}\left(\frac{N_{0}}{V}+x_{V}\right)^{2}-\left(\mu+\frac{v_{0}}{2 V}\right)\left(\frac{N_{0}}{V}+x_{V}\right) \\
+y_{V} \frac{N_{0}}{V}-\frac{\nu}{\sqrt{V}}\left(a_{0}+a_{0}^{*}\right)+z_{V} .
\end{array}
$$

As in the previous section, we consider the matrix of each $h_{V}$ in the basis of the $N_{0}$-eigenstates and extend it to $\ell_{2}(\mathbb{Z})$ with zero elements for negative indices. The nonvanishing matrix elements of $h_{V}$ are

$$
\begin{aligned}
& h_{V}\left(x_{V}, y_{V}, z_{V}\right)_{j j}= \\
& \frac{v_{0}}{2}\left(\frac{j}{V}+x_{V}\right)^{2}-\left(\mu+\frac{v_{0}}{2 V}\right)\left(\frac{j}{V}+x_{V}\right)+y_{V} \frac{j}{V}+z_{V}
\end{aligned}
$$

and

$h_{V}\left(x_{V}, y_{V}, z_{V}\right)_{j, j+1}=h_{V}\left(x_{V}, y_{V}, z_{V}\right)_{j+1, j}=-\nu \sqrt{\frac{j+1}{V}}$

with $j \geq 0$.

Each $h_{V}$ is still an unbounded operator. We can zoom in on different parts of its spectrum by employing different $V$-dependent right shifts in $\ell_{2}(\mathbb{Z})$ and taking the limit $V \rightarrow \infty$. (Left shifts would only generate the zero matrix.) Suppose that $x_{V} \rightarrow x, y_{V} \rightarrow y$ and $z_{V} \rightarrow z$. The limits $x, y$ and $z$ may not be arbitrary nonnegative numbers, e.g. $y=0$ for the mean-field model and $y=v_{0} x$ for the imperfect Bose gas. Choose a sequence $S_{V}$ of positive integers such that $s_{V}=S_{V} / V \rightarrow s$. For the matrix elements the shift corresponds to a change of variables, $j=m+S_{V}$, yielding $h_{V}\left(x_{V}, y_{V}, z_{V}, s_{V}\right)_{m m^{\prime}}=$ $h_{V}\left(x_{V}, y_{V}, z_{V}\right)_{m+S_{V}, m^{\prime}+S_{V}}$. When $V$ tends to infinity the limit of $h_{V}\left(x_{V}, y_{V}, z_{V}, s_{V}\right)_{m m^{\prime}}$ exists and defines a doubly infinite Jacobi matrix $h(x, y, z, s)$ with nonvanishing elements [19]

$$
\begin{aligned}
h(x, y, z, s)_{m m} & \equiv \chi(x, y, z, s) \\
= & \frac{v_{0}}{2}(s+x)^{2}-\mu(s+x)+y s+z
\end{aligned}
$$

and

$$
h(x, y, z, s)_{m, m+1}=h(x, y, z, s)_{m+1, m}=-\nu \sqrt{s} .
$$

Formally $h(x, y, z, s)$ is a one-dimensional tight-binding Schrödinger operator with a constant potential (3.7), governing the motion of a particle of mass $\sim 1 /|\nu| \sqrt{s}$ along the spectrum of $N_{0}$. Physically $h(x, y, z, s)$ is associated with the infinite volume limit of a microcanonical ensemble of bosons characterized by four intensive variables: the condensate density $s$, the density $x$ and energy density $-\mu x+v_{0} x^{2} / 2+z$ of the uncondensed particles and the density of the interaction energy, $\left(y+v_{0} x\right) s$, between the condensate and the uncondensed particles. Because of the symmetry breaking field, the energy density of the condensate is still an operator of diagonal and off-diagonal matrix elements $-\mu s+v_{0} s^{2} / 2$ and $-\nu \sqrt{s}$, respectively.

For $s>0$ the spectrum of $h(x, y, z, s)$ is purely absolutely continuous. As a set, it is the interval

$$
[\chi(x, y, z, s)-2|\nu| \sqrt{s}, \chi(x, y, z, s)+2|\nu| \sqrt{s}]
$$

that can be found by solving the generalized eigenvalue equation $h \psi=e \psi$ with the usual exponential ansatz $\psi(m)=\exp (i m \alpha)$ and choosing any real $\alpha$ in the interval $[0, \pi]$. The spectral point corresponding to $\alpha$ is

$$
e(x, y, z, s, \alpha)=\chi(x, y, z, s)-2 \nu \sqrt{s} \cos \alpha .
$$

In particular, the lower edge of the spectrum is

$$
\begin{array}{r}
g_{x y z}(\mu, \nu, s)=\frac{v_{0}}{2} s^{2}-\left(\mu-v_{0} x-y\right) s-2|\nu| \sqrt{s} \\
-\mu x+\frac{v_{0}}{2} x^{2}+z
\end{array}
$$

with two linearly independent solutions $\psi(m) \equiv 1$ and $\psi(m)=m$. The ground state energy density of $H(\mathbf{n})$ is obtained by minimizing the lower edge value with respect to $s$,

$$
\begin{array}{r}
\lim _{V \rightarrow \infty} E_{0}[H(\mathbf{n})] / V=e_{x y z}(\mu, \nu)=\min _{s \geq 0} g_{x y z}(\mu, \nu, s) \\
=g_{x y z}\left(\mu, \nu, s_{x y}(\mu, \nu)\right)
\end{array}
$$

where the minimizer $s_{x y}$ does not depend on $z[20]$. Inspecting the expression (3.10) we see that whenever $\nu \neq$ $0, s_{x y}>0$ for any value of $\mu$. Hence, $\partial g_{x y z}(\mu, \nu, s) / \partial s=$ 0 at $s=s_{x y}$, that is, $\sqrt{s_{x y}}$ is a positive root of a cubic polynomial.

We have seen in the previous section that in the overall ground state $\mathbf{n}=0$, therefore $x=y=z=0$ provides the overall ground state energy density:

$$
\begin{gathered}
e_{0}(\mu, \nu)=\min _{s \geq 0}\left\{v_{0} s^{2} / 2-\mu s-2|\nu| \sqrt{s}\right\} \\
\equiv \min _{s \geq 0} g_{0}(\mu, \nu, s)=g_{0}\left(\mu, \nu, s_{0}(\mu, \nu)\right) .
\end{gathered}
$$

Equation (3.12) agrees with Eq. (1.6). We could have found $e_{0}$ without knowing the ground state in finite volumes, by minimizing $e_{x y z}$ over nonnegative values of $x$, $y$ and $z$. 
To obtain Eq. (1.7) we make the following observations:

(i) Choosing $\nu \neq 0$,

$$
\frac{\partial e_{0}(\mu, \nu)}{\partial \nu}=\left[\frac{\partial g_{0}(\mu, \nu, s)}{\partial \nu}\right]_{s=s_{0}}+\frac{\partial s_{0}(\mu, \nu)}{\partial \nu}\left[\frac{\partial g_{0}(\mu, \nu, s)}{\partial s}\right]_{s=s_{0}}=\left[\frac{\partial g_{0}(\mu, \nu, s)}{\partial \nu}\right]_{s=s_{0}}=-2 \operatorname{sgn} \nu \sqrt{s_{0}}
$$

(ii) The ground state energy $E_{0}(\mu, \nu)$ of $H_{\nu}^{\mathrm{FD}}$ is a concave (even) function of $\nu$. Concavity comes from that of the thermodynamic potential $-\beta^{-1} \ln \operatorname{Tr} \exp \left\{-\beta H_{\nu}^{\mathrm{FD}}\right\}$ by letting the temperature go to zero. $\left(E_{0}(\mu, \nu)\right.$ is also an analytic function of $\nu$ in finite volumes; the singularity of $E_{0} / V$ at $\nu=0$ develops only as $V$ tends to infinity.)

(iii) The Hellmann-Feynman theorem yields the ground state averages

$$
\left\langle a_{0}\right\rangle=\left\langle a_{0}^{*}\right\rangle=-\frac{1}{2 \sqrt{V}} \frac{\partial E_{0}(\mu, \nu)}{\partial \nu} .
$$

(iv) According to an inequality known as Griffiths' lemma [21] and applied here to the sequence of concave functions $E_{0}(\mu, \nu) / V$ converging to $e_{0}(\mu, \nu)$,

$$
\begin{aligned}
& \frac{\partial e_{0}(\mu, \nu+0)}{\partial \nu} \leq \liminf _{V \rightarrow \infty} \frac{1}{V} \frac{\partial E_{0}(\mu, \nu)}{\partial \nu} \\
& \quad \leq \limsup _{V \rightarrow \infty} \frac{1}{V} \frac{\partial E_{0}(\mu, \nu)}{\partial \nu} \leq \frac{\partial e_{0}(\mu, \nu-0)}{\partial \nu}
\end{aligned}
$$

implying equalities in points where $e_{0}$ is differentiable. With (3.14) and (3.15) for $\nu \neq 0$

$$
\alpha_{0}=-\frac{1}{2} \frac{\partial e_{0}(\mu, \nu)}{\partial \nu}
$$

and substituting (3.13) into this equation we find Eq. (1.7).

$E_{0}(\mu, \nu)$ being a concave function also of $\mu$, Eq. (1.8) can be proved from

$$
\frac{\partial e_{0}(\mu, \nu)}{\partial \mu}=\left[\frac{\partial g_{0}(\mu, \nu, s)}{\partial \mu}\right]_{s=s_{0}}=-s_{0}
$$

by an identical argument. Because in the Hamiltonian $\mu$ is coupled to $N$ and not to $N_{0}$, we can use the result of the former section, that the ground state expectation value of $N_{\mathbf{k}}$ vanishes if $\mathbf{k} \neq 0$. Therefore

$$
\rho_{0}=-\frac{\partial e_{0}(\mu, \nu)}{\partial \mu}
$$

which together with (3.17) yields Eq. (1.8).

For $\mu>0$ by solving the cubic equation we find

$$
\sqrt{s_{0}}=2 \sqrt{-q} \cos \left(\frac{1}{3} \arctan \frac{\sqrt{-q^{3}-r^{2}}}{r}\right)
$$

with $q=-\mu / 3 v_{0}$ and $r=|\nu| / 2 v_{0}$. Expansion of $\sqrt{s_{0}}$ up to first order in $|\nu|$ yields

$$
\begin{aligned}
& \alpha_{0}=\operatorname{sgn} \nu\left[\sqrt{\frac{\mu}{v_{0}}}+\frac{|\nu|}{2 \mu}+f_{1} \nu^{2}\right] \\
& \rho_{0}=\frac{\mu}{v_{0}}+\frac{|\nu|}{\sqrt{\mu v_{0}}}+f_{2} \nu^{2} \\
& e_{0}=-\frac{\mu^{2}}{2 v_{0}}-2|\nu| \sqrt{\frac{\mu}{v_{0}}}-f_{3} \nu^{2}
\end{aligned}
$$

where $f_{i}(\mu, \nu)$ are smooth functions away from zero and bounded at $\nu=0$, and $f_{2}$ and $f_{3}$ are positive.

\section{GAP TO ONE-PARTICLE EXCITATIONS WITH A NONVANISHING MOMENTUM}

Let us return to Eq. (3.11). The minimum of $g_{x y z}(\mu, 0, s)$ is attained at $s=\left(\mu-\mu_{c}(x, y)\right) / v_{0}$ which is positive if $\mu>\mu_{c}(x, y) \equiv y+v_{0} x$. This is the condition to have a nonvanishing Bose condensate at $\nu=0$ in the ground state of $H(\mathbf{n})$ with $\mathbf{n}$ chosen so that $x_{V}(\mathbf{n}) \rightarrow x$ and $y_{V}(\mathbf{n}) \rightarrow y$, cf. Eq. (3.3). If $\nu \neq 0$ and $\mu \leq \mu_{c}(x, y)$, the ground state condensate density $s_{x y}$ is still positive but tends to zero together with $\nu$. Now the full density is $x+s_{x y}$. Properties (i)-(iv) listed in the former section remain valid, thus the order parameter and the condensate density in the ground state of $H(\mathbf{n})$ will respectively be

$$
\alpha_{x y}=-\frac{1}{2} \frac{\partial e_{x y z}(\mu, \nu)}{\partial \nu}=\operatorname{sgn} \nu \sqrt{s_{x y}}
$$

and

$$
\rho_{x y}=-\frac{\partial e_{x y z}(\mu, \nu)}{\partial \mu}-x=s_{x y} .
$$

We conclude that the relation $\alpha_{x y}(\mu, \nu)^{2}=\rho_{x y}(\mu, \nu)$ holds true for any $\mu$, provided that $\nu \neq 0$. For $\mu>$ $\mu_{c}(x, y), s_{x y}$ is given by the right-hand side of Eq. (3.19) with $q=-\left(\mu-\mu_{c}(x, y)\right) / 3 v_{0}$ and $r=|\nu| / 2 v_{0}$.

Substituting $s_{x y}$ into Eq. (3.10) we obtain the ground state energy density for $H(\mathbf{n})$. To prove the formula (1.11) this general expression is not needed. We set $\mathbf{n}_{\mathbf{q}}=1$ and $\mathbf{n}_{\mathbf{k}}=0$ for $\mathbf{k} \neq \mathbf{q}$. Then

$$
H(\mathbf{n})-H(0)=\varepsilon(\mathbf{q})-\mu+\left[v_{0}+v(\mathbf{q})\right] N_{0} / V .
$$


Averaging with the ground states of $H(\mathbf{n})$ and $H(0)$, respectively,

$$
\begin{aligned}
\varepsilon(\mathbf{q})-\mu+\left[v_{0}+v(\mathbf{q})\right]\left\langle N_{0}\right\rangle_{\mathbf{q}} / V \\
\leq E_{\mathbf{q}}-E_{0} \equiv E_{0}[H(\mathbf{n})]-E_{0}[H(0)] \\
\leq \varepsilon(\mathbf{q})-\mu+\left[v_{0}+v(\mathbf{q})\right]\left\langle N_{0}\right\rangle_{0} / V
\end{aligned}
$$

by the variational principle. In the present case $x=y=$ $z=0$, so both $\left\langle N_{0}\right\rangle_{\mathbf{q}} / V$ and $\left\langle N_{0}\right\rangle_{0} / V$ equal $\rho_{0}+O(1 / V)$. For $\mu>0$ we insert $\rho_{0}$ from (3.20) and obtain Eq. (1.11).

\section{EXTENSION TO POSITIVE TEMPERATURES}

Our analysis of microcanonical ensembles, especially equations (4.1) and (4.2) make it possible to extend the result (1.3) to thermal equilibrium states. Below we outline the argument leading to this conclusion.

In the microcanonical ensemble $\{x, y, z, s\}$ the condensate density is $s$, while the order parameter operator $\lim _{V \rightarrow \infty} a_{0} / \sqrt{V}$ is represented by a matrix $o$ with elements $o_{m, m+1}=\sqrt{s},-\infty<m<\infty$ and 0 otherwise. We can compute the mean value of $o$ in a generalized eigenstate $\psi$ of $h(x, y, z, s)$ by the formula

$$
\langle o\rangle_{\psi}=\lim _{M \rightarrow \infty} \frac{\sum_{-M \leq m \leq M} \psi^{*}(m)(o \psi)(m)}{\sum_{-M \leq m \leq M}|\psi(m)|^{2}} .
$$

Consider $\psi_{ \pm \alpha}(m)=e^{ \pm i m \alpha}, \psi_{\alpha, c}(m)=\cos m \alpha$ and $\psi_{\alpha, s}(m)=\sin m \alpha$, all belonging to the spectral point (3.9). Now $\langle o\rangle_{\psi_{ \pm \alpha}}=e^{ \pm i \alpha} \sqrt{s}$, while

$$
\langle o\rangle_{\psi_{\alpha, c}}=\langle o\rangle_{\psi_{\alpha, s}}=\cos \alpha \sqrt{s} .
$$

We want to treat the above mean values as limits of averages taken with eigenvectors of $H_{\nu}^{\mathrm{FD}}$. By a general property of one-dimensional second order difference equations, all eigenvalues of $H(\mathbf{n})$ are nondegenerate and therefore the eigenvectors are real. This property is inherited by $H_{\nu}^{\mathrm{FD}}$ if we disregard any possible accidental coincidence of eigenvalues belonging to different $\mathbf{n}$. So considering separate eigenvectors the relevant relation is (5.2) in the sense that for any sequence of eigenvectors $\psi_{l}$, the volume increasing with $l$, for all possible limits

$$
\lim \frac{1}{\sqrt{V}} \frac{\left\langle\psi_{l}\left|a_{0}\right| \psi_{l}\right\rangle}{\left\langle\psi_{l} \mid \psi_{l}\right\rangle}=\cos \alpha \lim \frac{1}{\sqrt{V}}\left(\frac{\left\langle\psi_{l}\left|N_{0}\right| \psi_{l}\right\rangle}{\left\langle\psi_{l} \mid \psi_{l}\right\rangle}\right)^{1 / 2}
$$

where $\alpha$ can assume any value. Note also that computing the average with the help of a density matrix we have to take a trace and then find the same outcome (5.2) or (5.3) whether we use the complex basis $\psi_{ \pm \alpha}$ or the real one, $\psi_{\alpha, c}, \psi_{\alpha, s}$.

Unless $\alpha=0$ or $\pi$, Eq. (5.3) is in conflict with Bogoliubov's hypothesis, supposing an asymptotic equality in the Schwarz inequality (1.2). The fact that Bogoliubov's suggestion proves nevertheless to be correct and we obtain Eq. (1.3) in thermal equilibrium is due to the strong equivalence of the grand-canonical and a suitably chosen microcanical ensemble: fixing $\beta=1 / k_{B} T, \mu$ and $\nu$ and taking the thermodynamic limit, the distribution of the intensive variables, $e, x, y, z$ and $s$ among them, becomes a Dirac delta. Let the energy density be $e_{\beta \mu \nu}$. Although there is a continuum of the parameter sets $\{x, y, z, s\}$ yielding $e(x, y, z, s, \alpha)=e_{\beta \mu \nu}$ for different values of $\alpha$, cf. (3.9), because of the degenerate limit distribution of these variables only a unique set will asymptotically dominate. With this set $e_{\beta \mu \nu}$ is attained as the minimum of $e(x, y, z, s, \alpha)$ with respect to $\alpha$ and $s$ because the density of states of each $H(\mathbf{n})$ has a maximum at the bottom of the spectrum of $H(\mathbf{n})$. Minimization singles out $\alpha=0$ or $\pi$, depending on the sign of $\nu$; see Eq. (3.10). In conclusion, $e_{\beta \mu \nu}=e_{x y z}(\mu, \nu)$ and the thermodynamic limits of $\left\langle a_{0}\right\rangle_{\beta \mu \nu} / \sqrt{V}$ and $\left\langle N_{0}\right\rangle_{\beta \mu \nu} / V$ are $\alpha_{x y}$ resp. $\rho_{x y}$, cf. Eqs. (3.11), (4.1) and (4.2), with $x=x(\beta, \mu, \nu)$, $y=y(\beta, \mu, \nu)$ and $z=z(\beta, \mu, \nu)$. This proves the validity of Eq. (1.3). Note that $\mu_{c}(\beta)$, the critical chemical potential of the grand-canonical ensemble satisfies the relation

$$
\begin{aligned}
\mu_{c}(\beta) & =\mu_{c}\left(x\left(\beta, \mu_{c}(\beta), 0\right), y\left(\beta, \mu_{c}(\beta), 0\right)\right) \\
& =y\left(\beta, \mu_{c}(\beta), 0\right)+v_{0} x\left(\beta, \mu_{c}(\beta), 0\right) .
\end{aligned}
$$

Recently Pulé and Zagrebnov [22] computed the thermodynamic limit of the pressure of the mean-field Bose gas in the presence of a gauge-symmetry breaking field. Their formula reads

$$
\begin{aligned}
p(\mu, \nu)= & \lim _{V \rightarrow \infty} \frac{1}{\beta V} \ln \operatorname{Tr} e^{-\beta H_{\nu}^{\mathrm{MF}}}=\frac{v_{0}}{2} \rho^{2}(\mu, \nu) \\
& +p_{\text {ideal }}\left(\mu-v_{0} \rho(\mu, \nu)\right)+\frac{\nu^{2}}{v_{0} \rho(\mu, \nu)-\mu}
\end{aligned}
$$

where $\rho(\mu, \nu)$ is the unique solution of the equation

$$
\rho=\rho_{\text {ideal }}\left(\mu-v_{0} \rho\right)+\frac{\nu^{2}}{\left(v_{0} \rho-\mu\right)^{2}} .
$$

Here $p_{\text {ideal }}(\mu)$ and $\rho_{\text {ideal }}(\mu)$ are respectively the pressure and density of the ideal Bose gas for $\nu=0$. They are welldefined if the chemical potential is negative. The authors prove that for $\nu \neq 0, \mu-v_{0} \rho(\mu, \nu)<0$ indeed. Because the pressure in any finite volume is a convex function of $\nu$, we can use Griffiths' lemma to obtain the order parameter from the relation $\alpha_{x y}=\alpha_{x 0}=(1 / 2) \partial p / \partial \nu$. We find $\alpha_{x 0}=\nu /\left(v_{0} \rho(\mu, \nu)-\mu\right)$, thus

$$
\rho_{x 0}=\alpha_{x 0}^{2}=\rho(\mu, \nu)-\rho_{\text {ideal }}\left(\mu-v_{0} \rho(\mu, \nu)\right) .
$$

Since $\rho(\mu, \nu)$ is the total density, we can identify the density of the uncondensed particles as

$$
x=\rho_{\text {ideal }}\left(\mu-v_{0} \rho(\mu, \nu)\right) .
$$

If $\mu<\mu_{c}(\beta)=v_{0} \rho_{\text {ideal }}(0), \alpha_{x 0}$ goes to zero with $\nu$ : in this case $\mu-v_{0} \rho(\mu, \nu)$ converges to a negative value, $\rho(\mu, 0)$ 
is the unique solution of the equation $\rho=\rho_{\text {ideal }}(\mu-$ $\left.v_{0} \rho\right)$. This latter holds true at $\mu=\mu_{c}(\beta)$ when $\mu_{c}(\beta)-$ $v_{0} \rho\left(\mu_{c}(\beta), \nu\right)$ tends to zero with a vanishing $\nu$, but slower than $\nu$. If $\mu>\mu_{c}(\beta)$, the convergence to zero becomes linear and thus the limit of $\alpha_{x 0}$ will be nonzero.

\section{SUMMARY}

In this paper we have rigorously established the equivalence of Bose-Einstein condensation and a spontaneous breakdown of the gauge symmetry in some simplified models of interacting bosons. In these models one retains only that part of the pair interaction diagonal in momentum representation. Equivalence has been obtained in the form of Eq. (1.3), that we found to hold true for any nonzero value of the symmetry breaking field, not only in the limit of a vanishing field. As a by-product, we have disproved the compressibility sum rule in the ground state of the one-dimensional mean-field Bose gas. Our method was to study certain microcanonical ensem- bles with a fixed density and energy density of uncondensed particles. In the thermodynamic limit these ensembles are still described by a family of operators, socalled Jacobi matrices, known from the one-dimensional tight-binding electron theory. The limit of infinite volume could be done on the many-body Hamiltonians because of their commuting with the occupation number operators of nonzero momentum states; this made it possible to reduce the problem to that of a single mode, $\mathbf{k}=0$. The result was first obtained in the ground state of the microcanonical ensembles and then extended to thermal equilibrium states by arguing with the strong equivalence of grand- and microcanonical ensembles.

\section{Acknowledgment}

I am indebted to Péter Szépfalusy for many helpful discussions and for providing me with a copy of Bogoliubov's original report. This work was supported by OTKA Grants T 042914 and T 046129.
[1] O. Penrose, Phil. Mag. 421373 (1951); O. Penrose and L. Onsager, Phys. Rev 104576 (1956)

[2] N. N. Bogoliubov, Quasi-averages in problems of statistical mechanics, Dubna report D-781 (1961), in Russian

[3] M. R. Andrews, C. G. Townsend, H.-J. Miesner, D. S. Durfee, D. M. Kurn and W. Ketterle, Science 275637 (1997)

[4] P. C. Hohenberg, Phys. Rev. 158383 (1967)

[5] M. Bouziane and Ph. Martin, J. Math. Phys. 171848 (1976). This paper gives a fully rigorous proof of Hohenberg's theorem.

[6] Bogoliubov in Ref. [2] made the suitable substitutions into his inequality and obtained the $1 / q^{2}$ singularity necessary to prove the absence of symmetry breaking in one and two dimensions. To arrive at this conclusion he only should have summed the resulting inequality over the allowed wave vectors $0<|\mathbf{q}|<q_{0}$ in finite volumes before letting $V$ tend to infinity (with $q_{0}$ fixed) and then $\nu$ to zero.

[7] F. J. Dyson, E. H. Lieb and B. Simon, J. Stat. Phys. 18 335 (1978)

[8] A. Sütő, J. Stat. Phys. 112375 (2003); J. Stat. Phys. 117301 (2004)

[9] N. N. Bogoliubov, J. Phys. USSR II, 23 (1947)

[10] J. Ginibre, Commun. Math. Phys. 8, 26 (1968)

[11] K. Huang, C. N. Yang and J. M. Luttinger, Phys. Rev. 105776 (1957); see also K. Huang, Statistical Mechanics, John Wiley \& Sons, 1963, Ch. 13

[12] D. J. Thouless, The Quantum Mechanics of Many-Body Systems, Academic Press, 1972

[13] T. C. Dorlas, J. T. Lewis and J. V. Pulé, Commun. Math.
Phys. 15637 (1993)

[14] L. Pitaevskii and S. Stringari, J. Low Temp. 88377 (1991)

[15] H. Wagner, Z. Phys. 195273 (1966)

[16] This inequality can be found in Bogoliubov's report [2] with a factor $1 / 8$ instead of $1 / 4$. The formula was rederived by L. P. Pitaevskii, JETP Lett. 45185 (1987) with the factor $1 / 4$. In the positive temperature version $E_{\mathbf{q}}-E_{0}$ is replaced by $2 k_{B} T$. Accordingly, in [2] the prefactor is $1 / 4$ in the first term of the lower bound, while the stronger result of Hohenberg [4] with $1 / 2$ at the same place also holds true.

[17] When taking the limit of $E_{\mathbf{q}}-E_{0}$ it is understood that $\mathbf{q}$ is replaced by a sequence $\mathbf{q}_{V} \rightarrow \mathbf{q}$ so that $\mathbf{q}_{V}$ is allowed for $V$.

[18] D. Pines and Ph. Nozières, The Theory of Quantum Liquids, Vol. I, W. A. Benjamin, New York, 1966, Ch. 2

[19] It can be shown that the convergence of $h_{V}\left(x_{V}, y_{V}, z_{V}, s_{V}\right)$ to $h(x, y, z, s)$ is in the strong resolvent sense. For a definition of this latter see $M$. Reed and B. Simon, Functional Analysis, Academic Press, New York, 1980

[20] Because of the convergence in the strong resolvent sense there is a sequence of eigenvalues of $H(\mathbf{n}) / V$ converging to $e_{x y z}$, and it is easily seen that $E_{0}[H(\mathbf{n})] / V$ cannot tend to a smaller value.

[21] R. Griffiths, J. Math. Phys. 51215 (1964)

[22] J. V. Pulé and V. A. Zagrebnov, J. Phys. A: Math. Gen. 378929 (2004) and arXiv:math-ph/0405044 